\title{
Dépenses et utilisation de soins de santé: Une revue de littérature théorique et empirique
}

\begin{abstract}
Jouilil Youness
Ingénieur d'Etat en Economie Appliquée-Statistique, Cadre de l'Observatoire National de Développement Humain, ONDH-Maroc Doctorant en Economie et Gestion, Faculté des Sciences Juridiques,

Economiques et Sociales, Kénitra, Maroc
\end{abstract}

\section{Lechheb Houda}

Professeure Habilitée, Faculté des Sciences Juridiques,

Economiques et Sociales, Kénitra, Maroc

\section{Ouakil Hicham}

Professeur Habilité, Faculté des Sciences Juridiques,

Economiques et Sociales, Kénitra, Maroc

Doi: 10.19044/esj.2018.v14n33p156 URL:http://dx.doi.org/10.19044/esj.2018.v14n33p156

\begin{abstract}
This research paper will review the literature on both important theoretical and empirical studies in health microeconomics. For this purpose, the first part defines the health insurance scheme and its main objectives. The second part presents the theoretical framework of the health demand, in particular the effect of health insurance on the consumption of health care. Part III presents examples of previous empirical studies that have examined the effect of coverage by a health insurance scheme. The last paragraph presents the role of the other dimensions and their effects on the individual demand for health care. More specifically, this paper aims, among other things, to answer these questions. Does health insurance improve the state of health? How does it affect health expenditure? What are the problems of setting up comprehensive medical coverage? What is the case for other socioeconomic and sociodemographic and socio-cultural variables? It has been found from the analysis that there is a strong relationship between insurance and access to care. This observation has been confirmed through a synthesis of most of the work dealing with this issue.
\end{abstract}

Keywords: Health expenditures, health insurance, health care utilization 


\section{Résumé}

Ce papier passera en revue sur les différentes études tant théoriques qu'aux empiriques réalisées en microéconomie de santé. A cet effet, la première partie définie le régime d'assurance maladie et ses principaux objectifs. La deuxième partie présente le cadre théorique de la demande de santé en particulier l'effet de l'assurance maladie sur la consommation de soins de santé. La troisième partie expose des exemples des études empiriques antérieures qui ont traité l'effet de la couverture par un régime d'assurance maladie. Le dernier paragraphe présente le rôle des autres dimensions et leurs effets sur la demande individuelle de soins de santé. Plus précisément, ce papier a pour objectif, entre autres, de répondre aux questions suivantes. L'assurance maladie améliore-t-elle l'état de santé ? Comment elle affecte les dépenses de santé ? Quels sont les problèmes liés à la mise en place d'une couverture médicale complète ? Qu'en est le cas pour les autres variables socio-économiques et sociodémographiques et socio-culturels? Il s'est avéré d'après l'analyse menée qu'il existe une forte relation entre l'assurance et l'accès aux soins. Ce constat a été confirmé à travers une synthèse de la majorité des travaux traitant cette problématique.

Mots clés: Dépenses de santé, assurance maladie, recours aux soins de santé

\section{Introduction}

La santé, comme composant du capital humain, est l'un des secteurs fondamentaux de développement et de croissance de toute économie et qui reste au centre des préoccupations des pouvoirs publics.

L'accès à la santé est un droit fondamental. Ainsi, pour garantir à toutes et à tous l'égalité d'accès aux soins, les pouvoirs publics instaurent un mécanisme dit «couverture médicale». Il a pour objectif de couvrir les risques et les frais de soins de santé inhérents à la maladie ou à l'accident. Toutefois, on constate une forte inégalité quant à l'accès aux soins de santé dans la plupart des pays surtout dans les pays en voie de développement. Quel est donc l'effet de l'assurance maladie quant à l'accès aux soins ? Est-elle le seul facteur qui affecte l'utilisation et les dépenses de soins ?

Ce papier de recherche passera en revue la littérature sur les études importantes tant théoriques qu'aux empiriques réalisées en microéconomie de santé. A cet effet, la première partie définira le régime d'assurance maladie et ses principaux objectifs. La deuxième partie présentera le cadre théorique de la demande de santé en particulier l'effet de l'assurance maladie sur la consommation de soins de santé. La troisième partie exposera des exemples des études empiriques antérieures qui ont traité l'effet de la couverture par un régime d'assurance maladie. Le dernier paragraphe mettra en exergue le rôle 
des autres dimensions et leurs effets sur la demande individuelle de soins de santé.

Plus précisément, ce papier a pour objectif, entre autres, de répondre à ces questions. L'assurance maladie améliore-t-elle l'état de santé ? Comment affecte-t-elle les dépenses de santé ? Quels sont les problèmes liés à la mise en place d'une couverture médicale complète ? Qu'en est le cas pour les autres variables socio-économiques et sociodémographiques et socio-culturels?

\section{Assurance maladie : Concepts et objectifs Définitions}

L'assurance maladie est un mécanisme par lequel une personne « dite assurée »se protège contre la perte financière causée par une maladie, un accident ou encore une invalidité. (Knight, 1921)

L'assurance maladie peut être définie encore comme un processus par lequel une personne se protège contre les pertes financières causées par un accident ou une invalidité afin d'améliorer l'utilisation des soins de santé et protéger les ménages contre l'appauvrissement des dépenses personnelles.

En effet, quand l'homme vivait seul ou dans des groupes familiaux primitifs, l'assurance médicale au sens formel du terme n'était pas si nécessaire puisque chaque famille ou groupe de familles s'occupait de ces proches du mieux qu'elle pouvait. Quand la vie en communauté est devenue plus complexe, les hommes ont reconnu la nécessité d'un système de protection qui leur permet de s'entraider en temps de crise et de maladie. Ainsi, les premiers régimes d'assurance ont été initiés et développés à partir de ce besoin (Kelly, 1951).

\section{Objectifs de la couverture par une assurance maladie}

L'assurance maladie garantit, pour les assurés et leurs ayants de droit, la couverture des risques et frais de soins de santé inhérents à la maladie ou l'accident et à la maternité. Ainsi, elle donne droit à la prise en charge des frais de soins curatifs, préventifs et de réhabilitation médicalement requise par l'état de santé du bénéficiaire.

La couverture par une assurance maladie est devenue une obligation en raison de la nature imprévisible des dépenses de soins de santé. En fait, puisque les personnes avancent dans l'âge, plus que la probabilité d'être atteinte par une maladie augmente. Ainsi, les individus ont une idée générale de leur besoin de services médicaux futurs. Toutefois, le montant exact qu'ils dépensent pour les soins de santé reste pour eux en grande partie incertain. (Asymétrie de l'information). Les dépenses de santé restent également beaucoup biaisées. Dans une telle situation, la possession d'une assurance maladie permet de protéger les individus contre l'éventuel dommage lié aux soins de santé. (Eichner, 1998) 
Le lien entre la couverture par une assurance maladie et l'état de santé est une relation de cause à effet. En fait, l'assurance maladie réduit significativement le coût du traitement supporté par le patient. Ainsi, l'utilisation de soins de santé sera plus élevée chez les personnes bénéficiant d'une assurance maladie que les non couvertes.

La plupart des recherches en microéconomie de santé approuvent que l'extension de la couverture médicale a un impact positif sur l'accès aux soins, l'utilisation des services, l'accessibilité économique des soins et la sécurité financière de la population en particulier pour les ménages à faible revenu. Ainsi, Il existe généralement une relation positive entre l'accès à l'assurance maladie et la consommation de soins de santé puisqu'elle augmente la quantité de soins de santé consommée. Van Doorslaer (2002) a montré qu'il existait une relation positive entre l'état de santé et la productivité au niveau individuel.

\section{Revue de littérature : Assurance maladie et demande de soins Revue théorique}

Multiples études ont été menées sur les différents aspects des services de santé et des domaines connexes.

Les études antérieures sur l'utilisation des services de soins de santé ont montré que l'assurance maladie influence positivement l'utilisation des services de santé. En effet, la possession d'une assurance entraîne plus d'options de financement, ce qui a un impact sur le choix du prestataire de soins de santé effectué par les assurés. (Pourezza J, 2007)

L'assurance maladie, comme les autres formes d'assurance, n'est pas à l'abri de problèmes. Les principaux problèmes liés à l'assurance maladie sont la sélection adverse, l'aléa moral et l'abus de l'assurance maladie.

Les paiements directs des soins de santé par les ménages peuvent avoir de graves conséquences sur l'accès aux services et la protection financière des personnes non assurées et par la suite sur leurs appauvrissements. En conséquence, de nombreux pays s'orientent vers un modèle de financement de la santé en regroupant les risques par le biais de l'assurance maladie.

Le partage des coûts, appelé également coassurance ou encore la franchise, est la partie de coût supportée par l'assuré à l'issu de chaque sinistre (Faulkner, 1960). L'application de ce principe exige que l'indemnité pour perte de salaire couvre la majeure partie de la perte, mais pas la perte totale.

De plus, Arrow, K (1963) a révélé que les soins partiels ou totaux permettent aux preneurs d'assurance d'avoir accès à un grand nombre de traitements de santé, en particulier les plus coûteux. En effet, l'abus des soins de santé peut être expliqué, non pas parce que les assurés sont davantage exposés à la maladie, mais plutôt parce qu'ils ont davantage recours aux soins morbides et aux actes de prévention. Ce phénomène est connu des spécialistes 
de la microéconomie de la santé par son risque moral qui entraîne une surconsommation des soins de santé et, partant, une perte de bien-être social.

Ainsi, une corrélation positive devrait exister entre le montant des dépenses de santé et le niveau de couverture. En fait, la couverture des risques implique une réduction des prix en cas de maladie, ce qui peut encourager l'assuré à consommer plus de soins. Le but de cet article est donc de tester et de valider l'hypothèse de l'aléa moral, selon laquelle les meilleurs assurés sont ceux qui consomment plus de soins de santé.

La mise en place d'un système d'assurance incite les assurés à consommer plus. De plus, il a montré qu'un taux de remboursement élevé peut pousser les gens à consommer plus de soins de santé (Pauly J.A., 1968). Pour limiter les comportements de surconsommation, Shavell (1979) a préconisé une couverture incomplète. En d'autres termes, une partie de la charge de soins doit être supportée par l'assuré afin de modérer ses dépenses de santé.

Claussat et Glaude (1993), utilisant un modèle d'équations simultanées, ont conclu que la couverture médicale avait un impact positif sur la probabilité d'utilisation des soins de santé. De plus, ils ont montré que l'assurance complémentaire est positivement corrélée à la demande de soins.

Rothschild et Stiglitz (1976) stipule que les personnes les plus risquées sont les plus disposées à souscrire une assurance maladie puisqu'elles savent que le montant qu'elles dépenseront pour les soins de santé sera supérieur à la prime qu'ils vont payer. Ce phénomène est appelé la sélection adverse.

Cependant, il existe une autre catégorie de personnes qui sont susceptibles de souscrire une assurance de santé. Ceux sont généralement celles qui attachent une grande importance à leur état de santé et à leurs finances. Ce phénomène est connu sur le marché de la micro assurance par la sélection positive. Il peut équilibrer la sélection adverse et permettre aux compagnies d'assurance de mutualiser les risques et par la suite de rester financièrement viable sans subventions.

Annear et al. (2006) stipule que les ménages qui se confronte à un choc sanitaire à court terme peuvent subir la pauvreté à moyen et à long terme. On parle à ce niveau des dépenses catastrophiques. En fait, des dépenses de soins de santé élevées peuvent pousser les familles à puiser leurs économies ou à les inciter d'emprunter auprès des banques ou à vendre leurs avoirs en particulier dans les pays en voie de développement. Toutefois, les ménages qui ne peuvent pas facilement emprunter auprès du marché bancaire, peuvent renoncer à des soins de grande valeur et de grande importance pour leurs états de santé et par la suite à chercher à des solutions alternatives (la médecine traditionnelle, par exemple).

Cependant, certains chercheurs malgré leur rareté (Kasper, J.P., Giovannini, T.-A. et Hoffman, C, 2000), (Le Fur, P. et Perronnin, M, 2003) (Raynaud, D, 2003) ont trouvé un résultat controverse et que la couverture par 
une assurance maladie avait un impact négatif ou nul sur l'état de santé. De plus, ils ont montré que l'état de santé des bénéficiaires est nettement moins bon que celui du reste de la population Française.

Il est à noter que la demande de soins de santé comporte certaines caractéristiques particulières qu'il importe de prendre en considération.

La demande de soins de santé est une demande dérivée de la demande de santé. En effet, les services de soins de santé ne sont pas demandés pour leurs caractéristiques propres, mais plutôt parce qu'on attend de ceux-ci un effet positif sur l'état de santé de l'intéressé. Ainsi, une diminution du prix des soins n'impliquera pas forcément une forte demande de soins. On parle à ce niveau d'une substituabilité limitée entre les soins de santé et les autres biens (Nyman, 2008).

Aussi, la demande de soins est une demande induite de la demande de soins de santé puisqu'elle passe le plus souvent par un prescripteur (médecin, pharmacien) qui détermine quels soins sont nécessaires et les médicaments à consommer et avec quelle quantité. La conséquence de cela est qu'une séparation, qui est habituellement faite, entre l'offre et la demande perd son efficacité dans le cas des biens de santé. Ainsi, l'offre sanitaire (publique ou privée) aurait un effet direct et immédiat sur la demande de soins de santé (Zukevas, 2014).

Enfin, les soins de santé sont des biens fortement hétérogènes. Il n'y a pas de remplacement possible entre la majorité des soins. Si l'on doit, par exemple, subir une opération de chirurgie vasculaire ou d'Oto-rhinolaryngologie, on ne peut pas la substituer tout simplement par des médicaments contre les maux de tête. La conséquence de cela est qu'il devient moins fondé de raisonner sur «les soins de santé » comme une catégorie homogène (Deaton et Muellbauer, 1980).

Il est à signaler que le concept de la demande de soins se diffère de celui de besoins de soins. Le premier est un concept descriptif et a pour finalité de représenter les comportements des individus et des ménages et leurs attitudes par rapport aux risques. Toutefois, le deuxième est normatif et qui s'examine par rapport à une pathologie ou une maladie donnée. Par exemple, quels seraient les soins qu'il faudrait faire lorsqu'un patient a une maladie donnée.

\section{Revue empirique}

$\mathrm{Au}$ niveau empirique, mesurer l'impact de l'assurance maladie sur l'utilisation ou les dépenses de soins est un exercice difficile à conduire puisque l'état de santé des individus est difficilement mesurable et parfois inobservable. Il existe néanmoins plusieurs méthodes pour 1'approcher. Le choix de l'approche conditionnera le modèle économétrique à préconiser. (Grossman M., 1972) 
La première et la seule expérience randomisée qui examine les effets de l'assurance maladie sur la santé des bénéficiaires a été réalisée entre 1974 et 1982 aux États-Unis nommée Expérience RAND Health Insurance Experiment (Joseph P., 1993). Cette expérience a étudié près de 4000 personnes dans 2000 ménages. Certaines familles ont été assignées au hasard à un régime de soins gratuit, tandis que d'autres ont reçu l'un des nombreux plans nécessitant des co-paiements variables $(10 \%, 20 \%, 40 \%)$. L'étude a révélé que les personnes affectées à un régime de partage des coûts recherchaient moins de traitement que celles bénéficiant d'une couverture complète.

L'assurance maladie a pour objectif principal d'améliorer l'accès aux soins de santé et de réduire les dépenses individuelles, en particulier pour les personnes à revenu limité. La mutualisation des risques du dispositif d'assurance maladie pourrait alors réduire les coûts des soins de santé et, par conséquent, augmenter la demande en services de santé.

Afin d'exposer le rôle principal de l'assurance maladie, plusieurs théories ont été utilisées. En effet, en utilisant un modèle en deux parties Ekman, B (2007) a prouvé que l'assurance maladie pouvait améliorer l'accès aux soins de santé et réduire les dépenses personnelles. En fait, le comportement de l'individu est perçu comme un double processus de décision de la demande de soins de santé. La première décision est la possibilité de voir un fournisseur de soins de santé et le second est la quantité de soins à obtenir.

Selon Deborah Stone (2008), la couverture par l'assurance maladie, en réduisant le prix des soins médicaux, incite les patients à obtenir davantage de soins de santé sans avoir à en payer le coût total, ce qui peut entraîner une réduction des activités de prévention.

La majorité des études empiriques ont mis en évidence une relation négative significative entre l'assurance maladie et les dépenses de santé (Jowett M., Deolalikar .A \& Martinsson .P. , 2004) et (Berkman, L .F., T. Glass, I. Brissette and T. E. Seeman, 2000). (Anderson, G, et Peter H., 2000) (1978) et (Grossman M., 1972) ont réalisé le premier travail sur les effets de l'assurance maladie dans les années 1900. Celles-ci ont montré que l'assurance maladie avait un effet considérable sur l'utilisation et les dépenses de santé.

Quelques années plus tard, Jowett al. (2004) ont montré que les personnes titulaires d'une couverture maladie sont plus susceptibles de faire appel à des services de soins de santé. En outre, ils ont révélé que l'absence d'assurance maladie avait un impact significatif sur la décision de consultation individuelle et l'utilisation des services de soins de santé. Berman (2001) a démontré le même résultat à l'aide de données égyptiennes.

Steyn K, et al, (2005) ont prouvé que l'assurance maladie peut améliorer l'accès des consommateurs aux produits pharmaceutiques. En effet, en comparant entre les assurés aux personnes non assurées au sein du même échantillon, ils ont trouvé que l'assurance était associée à une utilisation 
accrue des médicaments, à une réduction des obstacles financiers, à une utilisation plus rationnelle et à de meilleurs résultats en matière de soins de santé.

Dans le même ordre d'idée, la théorie économique de la microéconomie de santé affirme que l'assurance maladie a le potentiel d'augmenter l'utilisation des services de soins de santé. En effet, Zweifel et al. (2000) et Manning et Newhouse (1987) ont montré que l'assurance maladie peut augmenter la demande individuelle de soins médicaux. De plus, avoir une assurance maladie garantit aux assurés la stabilité de leur niveau de vie (Caussat Laurent, Raynaud Denis, 2004). De leur côté, Gardiol et al. (2003) ont analysé l'effet de l'assurance maladie complémentaire sur l'utilisation des soins de santé. Ils trouvent qu'avoir une couverture médicale complémentaire accroit la chance de consommer plus de soins médicaux ce que peut être justifié par le haut niveau de remboursement des assurés.

Selon Zweifel et Manning (2000) et par la mise en commun des risques entre assurés, la couverture maladie fait baisser le prix des soins de santé au moment de l'achat, ce qui entraîne une utilisation accrue des soins de santé. Dans le même temps, l'impact sur l'utilisation dépendra du niveau des prestations fournies et des circonstances individuelles, aux côtés de facteurs liés à l'offre (nombre d'hôpitaux, médecins, infrastructures, etc.) qui affectent les niveaux d'utilisation des services.

Toutes les études empiriques mentionnées dans cette partie ont été basées sur des modèles économétriques de choix discrets tels que le modèle logit multinomial, la régression linéaire simple, le probit binomial, la logistique multinomiale emboitée, le probit multinomial indépendant, les équations simultanées ou encore les modèles censurés.

\section{L'effet des autres dimensions}

La théorie de la demande de soins fournit une guide des déterminants de la fonction de demande. Cependant, elle reste imparfaite et non exhaustive puisqu'elle ne fournit pas d'indication sur l'effet de certaines variables, comme les variables reliées au statut socio-culturel (la religion, la coutume, les traditions, ... etc.). Ces dernières peuvent impacter fortement la consommation de soins. (Pauly, 2011)

Les travaux de recherche ont mis l'accent sur l'importance des caractéristiques individuelles et comportementales dans la détermination de l'état de santé de l'individu. Ces modèles se distinguent en fonction de l'importance qu'ils attachent à chaque facteur (Berkman, L .F., T. Glass, I. Brissette and T. E. Seeman, 2000). Ainsi, outre l'influence de la couverture sanitaire, les facteurs socio-économiques et sociodémographiques et socioculturels jouent également un rôle déterminant dans la décision d'accéder aux services de soins de santé. 
La littérature économique classe les déterminants les plus souvent utilisés en quatre catégories : déterminants économiques, déterminants liés à la santé, déterminants liés à l'accès (l'offre), et déterminants démographiques.

\section{Dimension économique}

Pour les déterminants économiques, on trouve le coût du traitement (approximé par la présence d'une couverture médicale), les indicateurs de niveau de vie ou de revenu (parfois approximés par les dépenses globales ou les biens durables et/ou les avoirs du ménage). Les indicateurs sur le statut dans l'emploi et la nature de l'occupation sont le plus souvent omis pour des raisons d'endogénéité.

\section{Coût du traitement}

L'élément essentiel qui impact fortement la décision du patient à recourir aux fournisseurs de services de santé est le cout du traitement. Des dépenses de santé élevées rendent les individus moins susceptibles d'utiliser les services de soins de santé. Ce qui les pousse à chercher ailleurs à d'autres alternatives. Andersen \& Newman (1973)

Paradoxalement, Majumder (2006) a signalé que le coût élevé du traitement augmente les chances d'utilisation de sources de services de santé modernes par les patients qui souffre de maladie grave. L'auteur explique ce résultat par le fait que les coûts élevés de la maladie indiquent la présence de maladies complexes dépassant la capacité des prestataires de services traditionnels.

\section{Revenu ou le niveau de vie de ménage}

L'autre conséquence importante est l'effet du revenu sur la consommation individuelle de soins. De nombreuses études empiriques suggèrent que lorsque les personnes deviennent plus riches, elles exigeront des soins plus nombreux et de meilleure qualité et des soins plus coûteux.

Tanti-Hardouin (1994) a révélé que la demande en matière de santé est corrélée au revenu (revenu des individus et revenu de l'État). De plus, Dong et ses collaborateurs (2002) ont montré à partir d'une étude empirique que le revenu des ménages, le sexe de l'individu et le niveau d'étude ont un impact significatif sur les dépenses de santé.

En plus, et d'après Gertler et Van Der Gaag (1990), les ménages à revenu élevé ont une probabilité plus élevée de se faire soigner que ceux des ménages défavorisés. De sa part, McCaw-Binns et al. (1995) a fait ressortir une liaison positive entre le recours aux soins prénataux et la profession de la femme enceinte.

Des travaux menés par (Birch, S., J. Eyles, and K. B. Newbold, 1993) ont montré que le revenu était un facteur non significatif pour l'utilisation des 
services de soins de santé. Le même résultat a été approuvé empiriquement par une étude réalisée en 2005 par Arcury.

Glanz, Rimer et Viswanath (2008) estiment que la décision des individus à recourir aux soins préventifs est très dépendante à leurs vecteurs de caractéristiques individuelles et de leurs conditions socioéconomiques. Parmi les facteurs clés du recours aux soins, on trouve les facteurs sociodémographiques (Age de l'individu, le sexe, la taille du ménage, le milieu de résidence, l'état matrimonial...Etc.), les facteurs socioéconomiques du ménage (Le revenu du ménage, l'éducation niveau de vie, ...Etc.), les facteurs socio-culturels (le niveau d'instruction du chef de ménage... Etc.)

\section{Dimension liée à l'accès et à l'offre de soins}

Ils varient considérablement entre les études considérées. Souvent, ces derniers sont omis, pour des raisons d'endogénéité.

\section{Accessibilité}

Les recherches d'Andersen et Newman (1973), de Kroeger (1983) et d'Ager et Pepper (2005) ont affirmé que l'accessibilité et la disponibilité des fournisseurs de services de santé est un élément crucial, déterminant et explicatif de l'utilisation de services de santé. Ainsi, la décision du patient de choisir un prestataire de soins de santé ou d'utiliser les services de soins alternative dépend principalement de la disponibilité physique du fournisseur de services dans sa région de résidence.

Plus encore, l'accessibilité ne se limite pas seulement dans la disponibilité physique du prestataire de service mais aussi aux coûts de déplacement associés, le temps alloué, le coût d'opportunité perdu du salaire journalier et la disponibilité des moyens de transport. Ce qui peut affecter le choix et l'utilisation du fournisseur (Buor, 2003).

\section{Qualité des services offerts}

La qualité de service perçue joue un rôle important dans l'utilisation des services de santé

Les recherches menées par Babakus et Mangold, (1992), Mariko, (2003) et (Kim et al. (2012) ont montré l'importance de la qualité perçue par les individus sur l'utilisation des services de santé. Ainsi, avec une perception accrue des services de santé, la probabilité d'utilisation de ces services augmente. Et vice versa.

\section{Dimension démographique}

Les variables liées à la démographie en particulier l'âge de l'individu, son sexe, son milieu de résidence, son statut matrimonial ou encore son statut d'éducation sont systématiquement intégrés dans les études empiriques de 
l'offre de soins et même parfois avec des variables d'interaction (interaction deux à deux en particulier entre l'âge et le sexe).

\section{Age}

L'âge est parmi les variables démographiques les plus susceptibles d'influencer la décision d'un individu d'utiliser les services de soins de santé.

Beaucoup d'études empiriques (Andersen RM, Newman JF, 1973) (1973) et (Kroeger, A., 1983) ont examiné l'effet de l'âge sur l'utilisation et les dépenses de soins de santé. Ils ont rapporté l'importance de cette variable en tant que facteur déterminant et explicatif de la demande de soins de santé.

En fait, plus la personne avance dans l'âge, plus ce que son état de santé se détériore. Ce qui l'incite à consommer plus de soins à l'âge avancée.

Anderson et al. (2000) ont montré que l'utilisation de services de soins de santé et les dépenses de soins sont plus élevées chez les enfants et les personnes âgées que chez les plus jeunes.

\section{Genre}

Parmi les variables démographiques qui influencent la consommation de soins, on y trouve le genre.

Les recherches empiriques effectuées par Andersen et Newman (1973), Kroeger (1983), Asada et Kephart, (2007) et (Brown, J. R. and A. Finkelstein, 2009) sur les pays développés ont montré que les femmes sont plus sensibles à leurs besoins en matière de santé, car elles accordent plus d'attention à leur état de santé en particulier à leurs santés physiques. Grossman (1972) a montré que les femmes consomment plus de soins que les hommes.

Toutefois, d'autres chercheurs ont constaté que le genre ne joue aucun rôle dans l'utilisation des services (Lahana E, Pappa E, Niakas D, 2011). Ils ont trouvé que cette variable n'est pas statistiquement significative.

L'état matrimonial et le nombre d'enfants sont également des déterminants clés de l'accès aux soins de santé.

\section{Milieu de résidence}

Le milieu de résidence est l'une des variables explicative du recours aux fournisseurs de soins de santé. Les études réalisées par les chercheurs tels que Andersen \& Newman (1973), Sudha et al. (2003) et Lahana et al. (2011) ont soulevé l'importance de l'environnement résidentiel dans le contexte d'utilisation des services de santé. Ils ont abouti à ce que les personnes appartenant à des zones urbaines et rurales présentent des perceptions différentes de l'utilisation des services de santé. 


\section{Niveau d'instruction}

Selon le modèle de Grossman (1972), une augmentation du niveau d'éducation augmente les produits marginaux des soins médicaux mais nécessairement avec la même proportion.

Les recherches réalisées par Andersen \& Newman (1973) et Kroeger (1983) ont conclu que l'éducation est une variable qui peut expliquer le recours aux des soins de santé.

En analysant les données empiriques, Hendryx (2002), Thind (2004), Asada et Kephart (2007) et Lahana, Pappa et Niakas (2011) ont remarqué que le niveau d'éducation influence fortement l'utilisation des soins de santé. Ainsi, les personnes les mieux éduquées étaient plus sensibles à leur santé et plus susceptibles d'opter pour les services de santé que leurs homologues les moins scolarisées. Plus encore, ils ont remarqué que l'éducation influence aussi le type de prestataire de services de santé utilisé par les patients. Pour des besoins similaires, les personnes les moins scolarisées ont plus chance à faire recourir aux services d'urgence que les personnes ayant faite des études universitaires. Ces derniers ont plus de probabilité d'utiliser les services de santé primaires financés par le gouvernement.

\section{Statut matrimonial}

Des études antérieures réalisées par Newbold (1993), Patil et al. (2002), Brown et al. (2009) Sato, (2012) ont étudié l'effet de l'état matrimonial sur l'utilisation de soins de santé. Ils affirment que les mariés surtout les femmes sont plus susceptibles d'utiliser les services de santé que les femmes célibataires. Ces résultats peuvent être justifiés par le changement du mode de vie de l'individu entre le célibat et le mariage surtout pour les femmes (grossesse, accouchement, maternité, etc.)

\section{Dimension liée à la santé}

Ils comprennent des mesures directes de l'état de santé (présence de maladie passagère ou chronique, de handicap, obésité), parfois complétés par des variables subjectives de l'état général de santé et de l'attitude par rapport aux risques. A titre d'exemples, on peut citer la variable type de maladie, l'indice de la masse corporelle, le comportement à risque (cigarette, drogue, alcool, ...etc.) (Ruhm, J. C., 2012)

Il est à signaler que les dimensions et les sous dimensions qui sont citées ci-dessous ne sont pas exhaustives et complètes. Il existe d'autres variables explicatives de la consommation de soins de santé qui ne sont pas parfois observables et qui ne peuvent pas être captées par les enquêtes ordinaires. 


\section{Conclusion}

L'accès aux soins de santé un facteur crucial et un résultat du développement humain. Au niveau microéconomique, un meilleur état de santé peut aider les individus et les ménages à sortir de la pauvreté, et accroitre leurs capacités productives.

Dans la vie quotidienne, les individus sont exposés à de différents risques (La maladie, les accidents, le chômage, les décès) qui impactent leur patrimoine. Ainsi, la maladie est un risque imprévisible et lorsqu'elle survient, elle a des résultats néfastes sur les patients et sur leur ménage d'origine. Ce qui rend les familles plus vulnérables à la pauvreté. En effet, le mauvais état de santé affectent le bien-être des familles à cause de la diminution de leur capacité productive, ce qui implique la perte de revenu et par la suite une forte probabilité d'exposition à la pauvreté et à la vulnérabilité surtout pour les indigents (Tabor, 2005).

Dans ce papier de recherche, nous avons analysés l'effet de la couverture par une assurance sanitaire de point de vue théorique.

Dans un premier temps, nous avons défini les concepts et les objectifs de l'assurance maladie. Une revue de littérature à la fois théorique et empirique traitant la causalité entre la possession d'une assurance et le recours aux soins d'une part, et celle-ci avec les dépenses de soins de santé d'autre part a été réalisée. Il s'est avéré d'après l'analyse qu'il existe une forte relation entre l'assurance et l'accès aux soins. Ce constat a été confirmé à travers une synthèse de la majorité des travaux traitant cette problématique.

En dernier lieu, un état des lieux a été fait sur l'ensemble des dimensions susceptibles d'influencer la consommation et le recours aux soins. Il souligne l'importance des dimensions socio-économiques, sociodémographiques, et les dimensions liées à la santé et les dimensions liées à l'accès et à l'offre de soins dans la détermination de la fonction de consommation de soins médicale.

\section{References:}

1. Andersen RM, Newman JF. (1973). Societal and individual determinants of medical care utilization in the United States. Milbank Memorial Fund Quarterly- Health and Society. 51(1):95-124.

2. Anderson, G, and Peter H. (2000). Population Aging : A Comparaison Among Industrialized Contries. Health Affairs, 191-203.

3. Angrist, J., \& Pischke, J. (2008). Mostly harmless econometrics : an empiricist's companion. Princeton University Press.

4. Annear PL, Wilkinson D, Men RC and Van Pelt M. (2006). Increasing access to health services for the poor: Health financing and equity in Cambodia. . Working Paper. Phnom Penh, Cambodia. 
5. Arrow, K. J. (1963). Uncertainty and the welfare economics of medical care. The American Economic Review, Vol LIII, No. 5, 941-973.

6. Asada, Y. and Kephart, G. (2007). Equity in health services use and intensity of use in Canada. Biomed Central Health Services Research, 7(41), 1-12.

7. Berkman, L.F., T. Glass, I. Brissette and T. E. Seeman. (2000). From social integration to health: Durkheim in the new millennium. Social Science and Medecine, 51,6, 843-857.

8. Birch, S., J. Eyles, and K. B. Newbold. (1993). Equitable Access to Health Care: Methodological Extensions to the Analysis of Physician Utilization in Canada. Health Economics 2, 87-101.

9. Boutaleb, A. (2011). Social Determinants and Health Equity in Morocco. Dans W. H. Organisation (Éd.), Background papers for the World conference on social determinants of Health. Rio de Janeiro.

10. Brown, J. R. and A. Finkelstein. (2009). The Private Market for LongTerm Care Insurance in the United States: A Review of the Evidence. Journal of Risk and Insurance 76(1), 5-29.

11. Caussat L. \& Glaude M. (1993). Dépenses médicales et couverture sociales. Economie et Statistiques, Vol. 265, 31-44.

12. Caussat Laurent, Raynaud Denis. (2004). La régulation de la demande de soins : le rôle de l'assurance maladie dans la formation de la consommation de biens et services de santé. In: Revue d'économie financière, $\mathrm{n}^{\circ} 76,2004$. La régulation des dépenses de santé, 129-151.

13. Deaton, A., \& Muellbauer. (1980). Economics and consumer behavior. Cambridge: Cambridge University Press.

14. Delattre, E., \& Dormont, B. (2003). Fixed fees and physician-induced demand: A panel data study on French physicians. Health Economics, $12,741-753$.

15. Dong H.,Kouyate, B.,Cairns, J., Mugisha, F., Sauerborn, R. (2002). Willingness to pay for community based insurance in Burkina Faso. Health Economics, 12, 849-862.

16. Eichner, M. J. (1998). The Demand for Medical Care: What People Pay Does Matter. The American Economic Review. Vol. 88, No. 2, Papers and Proceedings of the Hundred and Tenth Annual Meeting of the American Economic Association., 117-121.

17. Ekman, B. (2007). The impact of health insurance on outpatient utilization and expenditure: evidence from one middle-income country using national household survey data. Health Research Policy and Systems.

18. Feldstein, M. (1973). The welfare loss of excess health insurance. The Journal of Political Economy, 2551-280. 
19. Gardiol, G. e. (2003). Separating Selection and Incentive Effects: an Econometrics Study of Swiss Health Insurance Claims Data. . Working paper.

20. Gertler et Van Der Gaag. (1990). The Willingness to Pay For Medical Care : Evidence From Two Developing Countries. The Johns Hopkins University Press, Baltimore, MD.

21. Glanz, R. \& Viswanath. (2008). Health Behavior and Health Education: Theory, Research and Practice.

22. Grossman M. (1972). On the concept of health capital and the demand for health. Journal of Political Economy 80 , 223-225.

23. Joseph P. (1993). Newhouse and the Insurance Experiment Group. Free for All? Lessons from the RAND Health Experiment, Cambridge, Mass.: Harvard University Press.

24. Jowett M., Deolalikar .A \& Martinsson .P. . (2004). Health insurance and treatment seeking behaviour: evidence from a low-income country. Health Economics. 13:. 845-857.

25. Kasper, J.P., Giovannini, T.-A. et Hoffman, C. (2000). Gaining and Losing Health Insurance : Strengthening the Evidence for Effects on Access to Care and Health Outcomes. Medical Care Research and Review, vol. 57, n³, 298-318.

26. Knight, F. H. (1921). Risk, Uncertainty and Profit. New York: Harper and Row, 1965.

27. Kroeger, A. (1983). Anthropological and socio-medical health care research in developing countries. Social Science and Medicine 17,3, 147-161.

28. Lahana E, Pappa E, Niakas D. (2011). Do place of residence and ethnicity affect health services utilization? evidence from Greece. Int Journal Equity Health 10:, 10-16.

29. Lahana et al. (2011). Do place of residence and ethnicity affect health services utilization? evidence from greece. International Journal for Equity in Health, 10-16.

30. Le Fur, P. et Perronnin, M. (2003). L'état de santé des bénéficiaires de la couverture maladie universelle complémentaire en 2002. Questions d'économie de la santé IRDES, nº76.

31. Manning, W., Newhouse, J, Duan, N., Keeler, E., Leibowitz, A.\& Marquis, M.,. (1987). Health insurance and the demand for medical care:. Evidence from a randomized experiment. American Economic Review 77, 251-277.

32. McCAW-Binns A., L. G. (1995). Under-users of antenatal care: a comparison of non-attenders and late attenders for antenatal care, with early attenders. Social Science and Medicine, 40, 1003-1012. 
33. Nyman, J. (2008). Health Insurance theory : the case of the missing welfare gain. European Journal of Health economics, 9, 369-380.

34. Pauly J.A. (1968). The economics of moral hazard revisited : Comment. American Economic Review, Vol. 58.

35. Pauly, M. V. (2011). Insurance and the Demand for Medical Care. Dans S. Glied, \& P. Smith (Éds.), The Oxford Handbook of Health Economics. Oxford: Oxford University Press.

36. Pourezza J, S. A. (2007). Effect of supplemental enzyme on nutrient digestibility and performance of broiler chicks fed on diets containing triticale. Int J Poult Sci 6, 115-117.

37. Raynaud, D. (2003). Impact de la CMU sur la consommation individuelle de soins. Études et Résultats, DREES, n ${ }^{\circ} 22$. L'Harmattan, Paris.

38. Rothschild, M. \& Stiglitz, J.E. (1976). Equilibrium in Competitive Insurance Markets: An Essay on the Economies of Imperfect Information. Quarterly Journal of Economies, 90, 629-650.

39. Ruhm, J. C. (2012). The Economics of Risky Health Behaviors. Handbook of Health Economics, Volume 2.

40. Shavell. S. (1979). On Moral Hazard and Insurance. The Quaterly Journal of Economics, 93, 541-562.

41. Steyn K, Bradshaw D, Norman R, Bradley H, Laubscher R. (2005). The use of prescribed drugs for common chronic conditions in South Africa in 1998. Pharmaco-epidemiology and Drug Safety, 2005, 14(2):, 91-100.

42. Stone, D. (2008). Understanding Fairness and Efficiency. In Health Politics and Policy.

43. Sudha, S.S., Karthic, R., Naveen., Rengarmanujam, J.,. (2011). Anti hyperlipidemic activity of Spirulina platensisin Triton $\mathrm{x}-100$ Induced Hyperlipidemic Rats. Journal For Drugs and Medicines 3(2), 32-37.

44. Tanti-Hardouin N. (1994). Economie de la santé. Paris, Armand Colin, 268p.

45. Thind A. (2004). Health Service Use by Children in Rural Bihar. Journal of Tropical Pediatrics, Vol. 50, No. 3.

46. Van Doorslaer, E. K. (2002). Equity in the Use of Physician Visits in OECD Countries: has equal treatment for equal need been achieved? Measuring Up: Improving Health Systems Performance in OECD Countries, OECD, Paris, 225-248.

47. Zukevas, S. (2014). Health Care Demand, Empirical Determinants of. Dans A. Culyer (Éd.), Encyclopedia of Health Economics (pp. 343354). Oxford: Elsevier. 
48. Zweifel, P., Manning, W.G. (2000). Moral hazard and consumer incentives in healthcare. In: Culyer, A.J., Newhouse, J.P. (Eds.), . Handbook of Health Economics, vol. 1. Elsevier Science, Amsterdam. 